\title{
Integration of nanodiamonds with NV-centers on optical silicon nitride structures
}

\author{
Anna Elmanova ${ }^{1, *}$, Ilia Elmanov ${ }^{1}$, Sophia Komrakova ${ }^{1}$, Alexander Golikov ${ }^{1}$, \\ Javid Javadzade ${ }^{4,7}$, Vadim Vorobyev $^{3}$, Stephan Bolshedvorskii ${ }^{3,4}$, Vladimir Soshenko ${ }^{3}$, \\ Alexey Akimov ${ }^{3,7,6}$, Vadim Kovalyuk ${ }^{1,2}$, and Gregory Goltsman ${ }^{1,2,5}$. \\ ${ }^{1}$ Moscow State Pedagogical University, 119991 Moscow, Russia \\ ${ }^{2}$ Zavoisky Physical-Technical Institute of the Russian Academy of Sciences, 420029 Kazan, Russia \\ ${ }^{3}$ Lebedev Physical Institute, 119991 Moscow, Russia \\ ${ }^{4}$ Moscow Institute of Physics and Technology, 141700 Dolgoprudny, Moscow region, Russia \\ ${ }^{5}$ National Research University Higher School of Economics, 101000 Moscow, Russia \\ ${ }^{6}$ Texas A \& M University, TX 77843, USA \\ ${ }^{7}$ Russian Quantum Center, 143025 Moscow, Russia
}

\begin{abstract}
In this work we had developed optical structures from silicon nitride for further integration of the nanodiamonds containing NV-centers with them. We have introduced method of the nanodiamonds solution application on the substrates. The work has practical meaning in nanophotonics sphere and in development of optical devices with singlephoton sources.
\end{abstract}

One of the main targets of nanophotonics is development and application of integral optical circuits for creation of the on-chip devices that can be used in transmission and processing both classical and quantum signals. For the quantum optical micro-circuits there is an unsolved problem of integration of all the components, including the source, logical elements and detectors. Application of nanodiamonds with NV-centres as single-photon sources is very perspective due to the advantages, such as a possibility to work with a room-temperature and a big time of conservation of the quantum state [1].

For integration of the nanodiamonds (solution with affiliated $\mathrm{COOH}$ group and NV centers contained) were used substrates of $\mathrm{Si}(525 \mu \mathrm{m})$ with thermally grown oxide $(2 \mu \mathrm{m})$ and $\mathrm{Si}_{3} \mathrm{~N}_{4}(200 \mathrm{~nm})$ on top. Thicknesses of dielectric layers provide the most efficient outcome of the light through the diffraction couplers of NV-centers radiation.

On a Fig. 1 (a) there is a diagram of the waveguide structure developed. Apodised diffraction grating couplers with period $542 \mathrm{~nm}$ and fill-factor 0.4 were picked up for the effective input and output of the radiation on the wavelength $680 \mathrm{~nm}$. The structure was divided on layers, corresponding to different doses for electron-beam lithography for the proximity effects correction using NanoMaker. The first stage of production was photolithography, evaporation of Ti/Au and lift-off in acetone, were formed golden signs which were used for the superimposition on the further stages with e-beam lithography.

\footnotetext{
* Corresponding author: anna.elmanova.belskaya@ gmail.com
} 

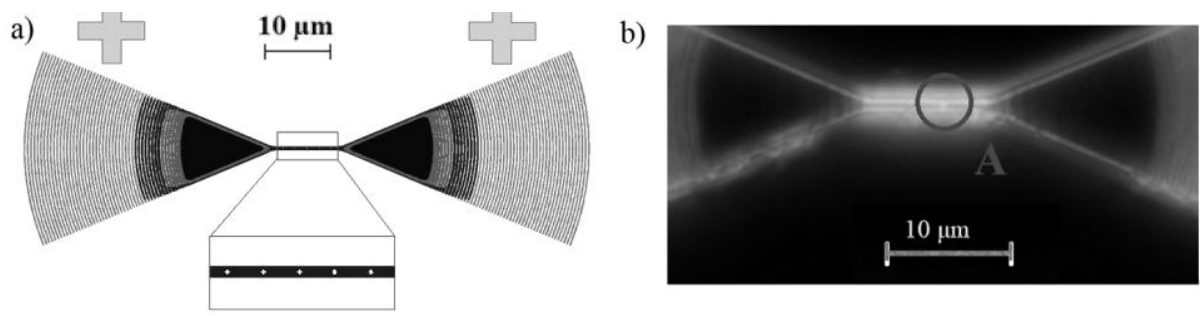

Fig. 1. Structures of $\mathrm{Si}_{3} \mathrm{~N}_{4}$ for the integration of nanodiamonds. (a) Schematics of the nanophotonic device with two diffraction grating couplers, waveguide, golden markers and holes for nanodiamonds. (b) Microphotograph of nanophotonic structure in a dark field. Structure after application of nanodiamonds and erasing of the resist. Letter "A" is put near some nanodiamond-like spot.

The next stage produced the nanophotonic device with electron-beam lithography and plasma etching in $\mathrm{CHF}_{3}$ atmosphere. Then the waveguides were provided with 5 holes each in a positive electronic resist PMMA 3\%, $200 \mathrm{~nm}$ diameter with e-beam lithography.

Due to the studied articles [2-4] was developed a method of nanodiamonds integration with nanophotonic structure. For separation of the nanodiamonds the solution was put into UV bath for 3 hours. $25 \mu$ of the solution were poured on the sample put on the hot-plate at temperature of $30-35^{\circ} \mathrm{C}$. For better adhesion there IPA was used with the solution. After heating the hot-plate to $65-70{ }^{\circ} \mathrm{C}$ the solution evaporated. Lift-off in acetone erased electronic resist, leaving nanodiamonds in the holes. In optical microscope were observed some pieces that looked like nanodiamonds. Measurements in AFM (NT-MDT) showed some bunches of matter, left after lift-off on the holes' places. It's not clear if the bunches are single nanodiamonds or small groups of them and are there any NV-centers. Further should be measured the correlation function of $2^{\text {nd }}$ order $g^{2}(\tau)$ on confocal laser scanning microscope, that will give us the clear information about the bunches.

Our research introduces a simple way of realization of the nanodiamonds integration with optical structures on $\mathrm{Si}_{3} \mathrm{~N}_{4}$.

The research was performed with the help of Ministry of Education and Science of the Russian Federation (convention № 14.586.21.0063, unique identificatory RFMEFI58618X0063).

\section{References}

1. I. Aharonovich, S. Castelletto, D.A. Simpson et al. Diamond-based single-photon emitters. Rep. Prog. Phys., 74(7), 076501 (2011).

2. S.I. Bogdanov, O.A. Makarova, A.S. Lagutchev A.S, D. Shah, et al, Deterministic integration of single nitrogen-vacancy centers into nanopatch antennas. (2019).

3. M. Kianinia, O. Shimoni, A. Bendavid, A. Schell, et al, Robust, directed assembly of fluorescent nanodiamonds. (Nanoscale, 8, 18032-18037, 2016).

4. J. Holzgrafe, J. Beitner, D. Kara, H.S. Knowles, M. Atatüre, Error corrected spin-state readout in a nanodiamond. Npj Quantum Information 5, 13 (2019). 